\title{
Visualizing electromagnetic fields at the nanoscale by single molecule localization
}

\author{
Christian Steuwe $^{\mathrm{a}, \mathrm{b}}$, Miklos Erdelyi ${ }^{\mathrm{a}}$, G. Szekeres ${ }^{\mathrm{c}}$, M. Csete ${ }^{\mathrm{c}}$, Jeremy J Baumberg ${ }^{\mathrm{b}}$, Sumeet \\ Mahajan $^{* \mathrm{~d}}$ and Clemens F. Kaminski*a \\ ${ }^{a}$ Department of Chemical Engineering and Biotechnology, University of Cambridge, \\ New Museums Site, Pembroke Street, Cambridge, CB2 3RA, UK \\ ${ }^{b}$ Nanophotonics Centre, Cavendish Laboratory, University of Cambridge, \\ J J Thomson Avenue, Cambridge, CB3 OHE, UK \\ ${ }^{\mathrm{c}}$ Department of Optics and Quantum Electronics, University of Szeged, H-6720 Szeged, \\ Dóm tér 9, Hungary \\ ${ }^{d}$ Institute for Life Sciences and Department of Chemistry, University of Southampton, University \\ Road, \\ Southampton, SO17 1BJ, UK \\ Corresponding authors: SM (s.mahajan@soton.ac.uk), CFK( $\underline{\text { cfk23@cam.ac.uk })}$
}

Coupling of light to the free electrons at metallic surfaces allows the confinement of electric fields to sub-wavelength dimensions, far below the optical diffraction limit. While this is routinely used to manipulate light at the nanoscale ${ }^{1}$, in electro-optic devices ${ }^{2}$ and enhanced spectroscopic techniques ${ }^{3-6}$, no characterization technique for imaging the underlying nanoscopic electromagnetic fields exists which does not perturb the field ${ }^{4,7}$ or employs complex electron beam imaging ${ }^{8,9}$. Here, we demonstrate the direct visualization of electromagnetic fields on patterned metallic substrates at nanometer resolution, exploiting a strong 'autonomous' fluorescence-blinking behavior of single molecules within the confined fields allowing their localization. Use of DNA-constructs for precise positioning of fluorescence dyes on the surface induces this distance-dependent autonomous blinking thus completely obviating the need for exogenous agents or switching methods. Mapping such electromagnetic field distributions at nanometer resolution aids the rational design of nanometals for diverse photonic applications. 
Keywords: Super-resolution, single molecule localization, plasmons, nanostructures, surface-enhanced

Nanophotonics has evolved into a hugely interdisciplinary field at the interface of optics, materials and nanoscience, with many applications in chemistry and biology ${ }^{2}$. Therefore, engineering well-defined nanostructured surfaces, which can sustain surface plasmon modes, is extremely important in technological applications such as enhancement of Raman scattering to permit the detection of single molecules ${ }^{10}$, or to obtain reproducible characteristics for quantitative diagnostics ${ }^{11}$. Usually, the morphological characterization of such nanostructures is performed with scanning electron microscopy techniques ${ }^{12}$. However, only a few techniques allow the measurement of electromagnetic field distributions of plasmonic modes with nanometer resolution ${ }^{8,13}$ without introducing field distortion. As a result, finite- and boundaryelement simulations are widely used instead to predict field distribution on plasmonic surfaces, but this is associated with significant uncertainties and limitations. Simulation results are algorithm dependent and can only predict fields for idealized structures, which are free of imperfections, a situation never achieved in practice $^{14}$.

Here we present a new technique, surface-enhanced localization microscopy (SELM), which does not suffer these limitations and exploits the plasmonic enhancement of fluorescence combined with single molecule localization microscopy to resolve optical fields across nanostructured metal surfaces with $20 \mathrm{~nm}$ resolution ${ }^{15,26}$ a . We use a simple labelling technique to precisely position robust fluorescent dyes on gold surfaces via a DNA scaffold and show that this leads to 'autonomous' blinking of the fluorophores. Unlike the conventional photochemical

${ }^{a}$ To evaluate this resolution metric from the actual image data described in this paper we the density estimation approach as explained in full detail in reference 26 was followed. 
approaches to induce blinking of fluorophores for super-resolution microscopy externally through reagents or the photoactivation/ excitation with a second laser wavelength ${ }^{16-18}$, we demonstrate here that the photodynamic response of standard fluorophores is considerably modified in the vicinity of nanostructured metal surfaces. The effect generates strong 'autonomous' blinking of conventional fluorophores which is sufficient to localize them at high resolution. Hence, super-resolution imaging by single molecule localization becomes possible without any requirement for chemical or optical control of fluorescent on- and off-states. Furthermore, by exploiting this autonomous switching, we are able to remove static interference efficiently, which otherwise impedes the correct localization of single emitters. We demonstrate that our powerful technique allows the mapping of nanoscopic EM field patterns in exemplar plasmonic structures, such as: Klarite ${ }^{\circledR}$, featuring a pyramidal pit structure as shown in Figure $1 \mathrm{a}^{19}$; nanovoids, 'dish-like' structures with variable diameter $D^{20}$ (Figure $1 \mathrm{~b}$ ); and random scratches on planar metal films. We find good agreement between experimental results of field distributions with the data obtained from finite element modelling. The simplicity of the SELM technique paves the way for its utilization in rational design of nanomaterials for diverse photonic applications including plasmon-enhanced fluorescence spectroscopy.

In this work we utilize fluorescent dyes immobilized on metallic surfaces for demonstrating the SELM technique. Unlike Raman scatterers, fluorescent molecules can suffer from non-radiative electron transfer in the vicinity of metal surfaces and this leads to quenching ${ }^{24,25}$. Thus, the maximum fluorescence emission is observed at a distance away from the surface due to the competition between non-radiative decay and field enhancement. In order to determine this distance empirically, we use dyes attached at different distal positions on double-stranded DNA (dsDNA) scaffolds (see supporting information). The fluorescent molecule is attached to a 
'probe strand' at a position near the 5' end, which binds to a 'surface strand' with a complimentary sequence (Figure 1c). The surface strand is covalently attached to the metal. Using cyanine dyes, Cy5 and Hilyte 647 , the optimal distance was found to be $\sim 20 \mathrm{~nm}$, corresponding to attachment of the dye at the 53rd base-pair position above the surface. The dyes have excitation and emission maxima at 630 and $647 \mathrm{~nm}$, respectively. A $642 \mathrm{~nm}$ laser was used for excitation, which is not only in resonance with the dyes but also with plasmons sustained on Klarite ${ }^{\circledR}$ and nanovoids. The plasmon resonance in Klarite ${ }^{\circledR}$ is very broad $>600 \mathrm{~nm}$ while nanovoids have stronger, tunable (size and thickness-dependent) although less broad resonances. These plasmonic properties of Klarite ${ }^{\circledR}$ and nanovoid surfaces have been studied in detail earlier $^{19,21-23}$. The fluorescence was detected at around $690 \mathrm{~nm}$. Figure 1d, shows a wide-field fluorescence image of Klarite $\AA$, a commercially available substrate used for surface-enhanced Raman scattering (SERS). Strong fluorescence from Hilyte647 is visible in area IV, where the fluorescent molecules are attached with $20 \mathrm{~nm}$ linkers to the gold-coated pyramidal pit-structured surface. In contrast, regions I, II, and III, shown in the same image, exhibit only very dim (20-50 times less intense) fluorescence signals, originating from either non-structured Pt- (II) or Au(III) coated regions or Klarite coated with Pt (I). Only region IV supports plasmons, is brighter than the other regions and thus clearly demonstrates their role on fluorescence signal enhancements. 

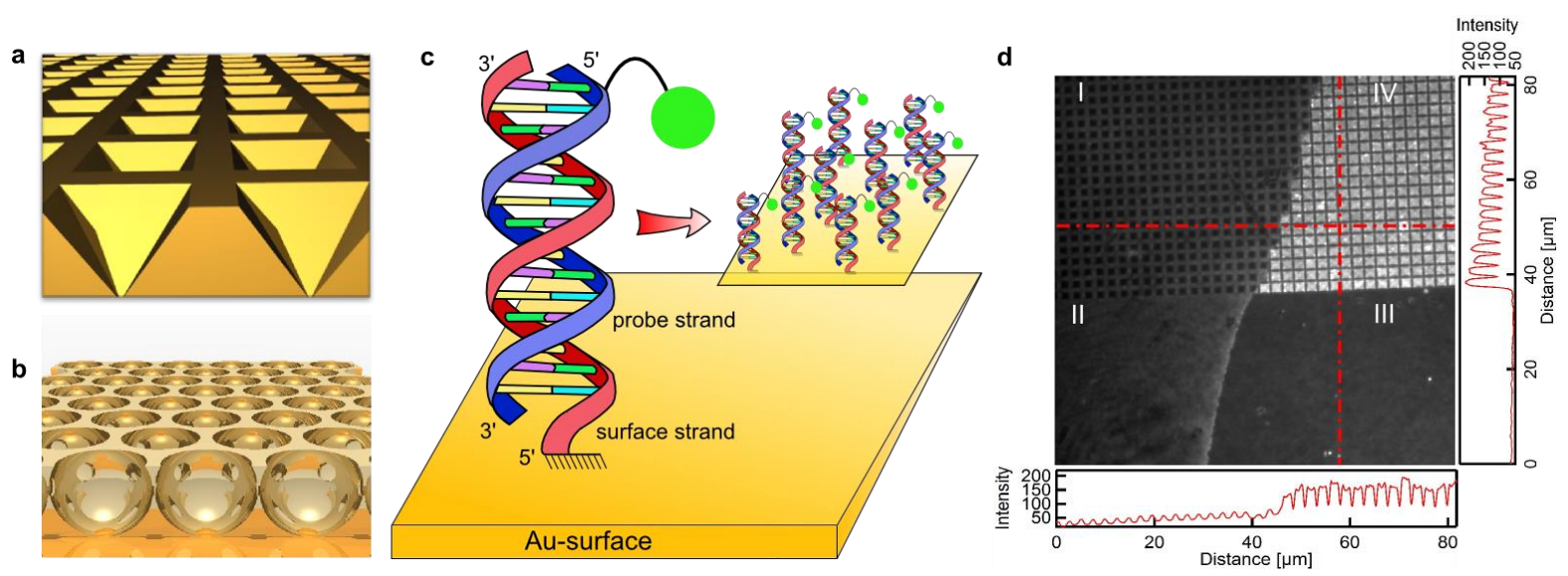

Figure 1: a, b) Schematic representations of Klarite ${ }^{\circledR}$ and nanovoid structured surfaces respectively. c) Sketch of the labelling technique involving two complementary DNA strands. The 'surface strand' is attached to the surface by a thiol bond; the 'probe strand' carries the dye molecule, here near its 5' end. d) Wide-field fluorescence image of a surface structure containing: (I) a platinum (Pt) plated pyramidal pit region, (II) a plain (non-structured) Pt plated region, (III) a plain Au coated region and (IV) an Au-coated Klarite surface. All regions were labelled with Cy5 positioned $\sim 20 \mathrm{~nm}$ above the surface (as in c). Only region IV supports surface plasmons, leading to the dramatically enhanced fluorescence signal seen. The red dash-dotted lines indicate sections along the surface for the two intensity profiles along the vertical and horizontal directions. The intensity profiles clearly show the enhancement in region IV compared to regions I and III.

For super-resolution imaging via single molecule localization one takes a large number of fluorescence images with sparsely distributed, blinking molecules and superimposes the reconstructed molecule positions. This then permits the structure of a labelled object to be resolved at nm precision ${ }^{17}{ }^{26}$. In our case, the dye density on the surface was controlled via the concentration of the probe strand. Incubating the surface for 10 min with a concentration of 100 $\mathrm{nM}$ of dye labelled probe and incubating with the surface for 10 min resulted in less than $\sim 15$ fluorescently active molecules per image frame (see supporting information, figure S4). This was suffucient to permit the precise localization of individual fluorescent spots. We made sure that the surface was completely covered with 'surface strands' and allowed the 'probe strands' to 
attach randomly to avoid artefacts during imaging that might otherwise arise from fluorophore density variations (see Materials \& methods in supporting information).

For SELM (surface enhanced localization microscopy) on Klarite, image stacks of up to 10000 frames, each with $20 \mathrm{~ms}$ integration time, were acquired. The Hylite 647 dye was offset by 20 $\mathrm{nm}$ from the surface using the dsDNA scaffolding scheme (above). Intriguingly, we observed very strong fluorescence blinking of the dye (see Video 1 and 2 of the supporting information). In our case this was observed in the absence of any photoactivation or addition of an external agent such as an aliphatic thiol, which is typically needed for stochastic optical reconstruction microscopy ${ }^{27}$. In addition, the photobleaching rate of the dye was drastically reduced (see supporting information and videos). All these attributes are advantageous in the context of single molecule super-resolution imaging with SELM and they are evidence of the dramatic modification of the dye properties in the proximity of the metal environment. In addition to dye fluorescence, a plasmon-enhanced gold photoluminescence was also observed. However, this static (non-blinking) background was removed effectively with a subtraction algorithm for improved localization (fitting) of the blinking observed from the dye (see the supporting information and Figure S4). It is also pointed out that the blinking behavior was not specific to Hilyte647 only and was observed with Cy5 as well (data not shown); however, the dependence of molecular structure on the blinking behavior remains to be investigated.

Figure 2a shows the SELM images on Klarite, which is an array of square pyramidal micro-scale pits. Features within individual pits are clearly resolved, while the conventional bright field image only shows coarse structures. Features observed inside each pit resemble one another across the surface - an indication of the high reproducibility of the Klarite substrate geometry from pit to pit. In a highly magnified view (Figure $2 b$ ), however, finely distributed details of the 
generated surface enhanced fluorescence (SEF) are revealed. We attribute the 'grainy' appearance of the monitored fields to the variable, uncontrollable surface roughness on the nanoscale, the latter borne out by SEM images as shown in Figure $2 \mathrm{~g}$.
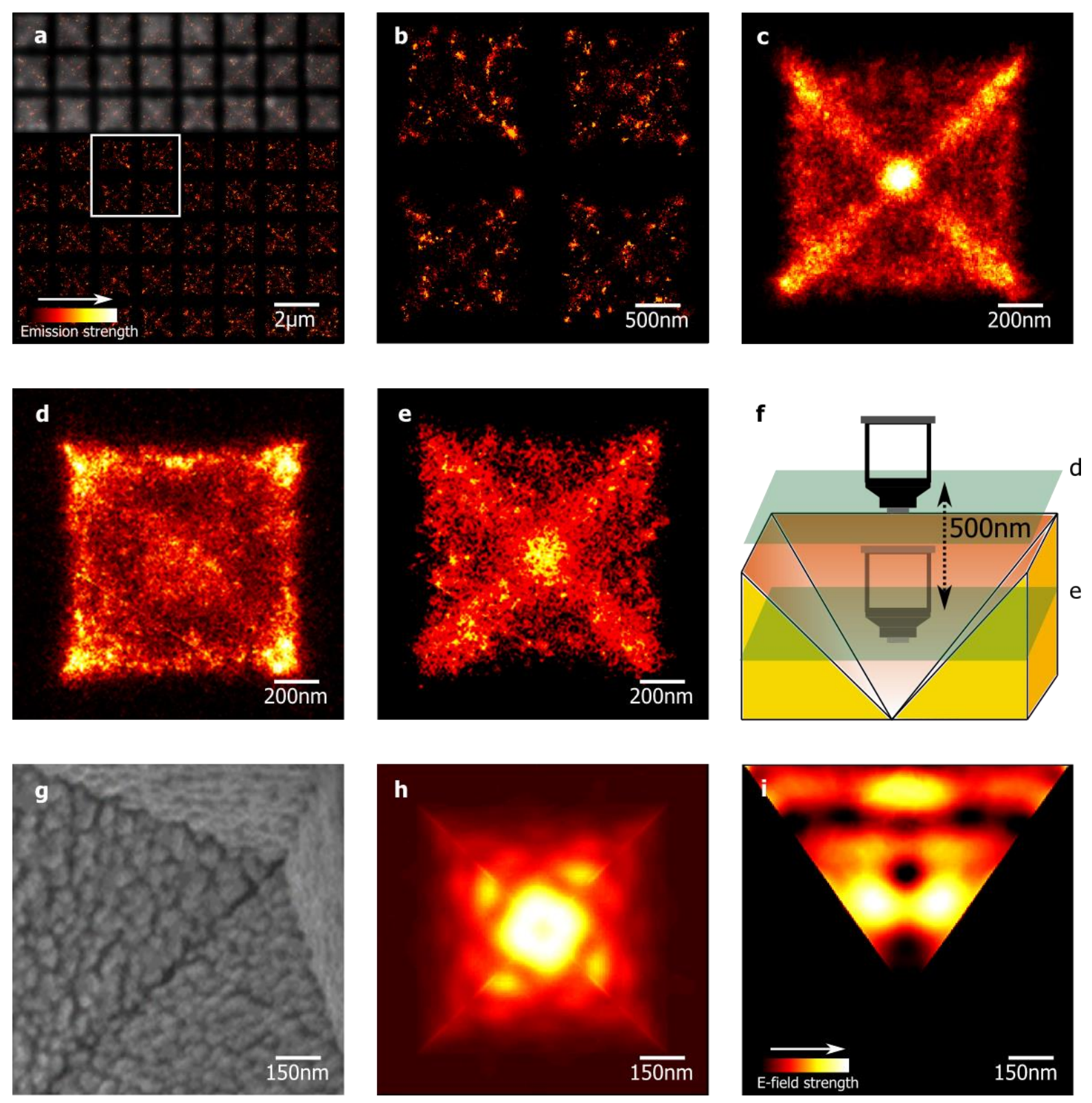

Figure 2: a) Reconstructed SELM image of a Klarite substrate (red regions) and conventional, diffraction limited, wide field fluorescence image (overlaid as greyscale image in the top third of panel). Whilst the bright field image sees the coarse periodic structure of Klarite, SELM clearly resolves individual features inside the pits; the emission 
strength of individual localizations is color-coded. In b), a higher magnification view is presented corresponding to the white inset shown in a). c) Overlay image containing SELM reconstructions from 60 pits of a), revealing the strong enhancement along the edges and towards the bottom tip of the Klarite structure. The flat sides of the pit are less active. d, e) SELM images at different focal heights inside a Klarite pit which differ by $500 \mathrm{~nm}$ as indicated in f). Images show slightly more granular signals than in c) as averages were taken over 25 pits only and the influence from the uncontrollable surface roughness is stronger. g) Scanning electron microscopy picture at 20 degrees angle off the surface normal of a Klarite pit. The surface roughness is clearly visible from the image. h, i) Finite element simulations of the electric fields inside a Klarite pit, see also the supporting information. h) Vertically integrated view showing strong modes along the angled trough edges. i) Vertical cut through a Klarite pit.

An average over SELM images of many pits may therefore serve as an approximation to an idealized Klarite geometry, which features ultraflat surfaces. Strikingly, in the SELM image shown in Figure 2c, reconstructed by overlaying 60 different pits, intensities along the angled troughs increase much more than those along the walls of the pit and therefore appear highlighted in the images. Strong field modes are evident towards the bottom tip of the pit structure, as well along the angled edges. We note that these observations are highly reproducible for different Klarite samples. The other signals distributed in the pit remain at their original intensities but become more uniformly distributed within the frame, therefore confirm that they are stochastic and originate from the enhanced SEF signals due to the variable surface roughness. The SELM mapping thus highlights that although the geometry is key to generating field enhancements the surface roughness plays an important role in the overall field enhancements that occur in Klarite $^{28}$. For comparison with the SELM image in Figure 2c, a conventional widefield image was also reconstructed by overlaying the same 60 pits (see the supporting information, Figure S5). It is clear that the resolution of SELM is dramatically higher than that 
achievable with conventional wide field imaging. This demonstrates the ultra-high sensitivity of SELM to visualize differences and stochastic variations of field patterns at ultra-high resolution in plasmonic structures. In Klarite we observe plasmons that appear as bright 'hot' spots on SELM images, which are representative of field enhancements caused not only by the large-scale pit geometry but also by nanoscale surface defects. These features are normally not visible in simulations which cannot take surface roughness into account. They are also not resolved by traditional imaging methods which probe averaged distributions at low resolutions.

To probe the plasmon fields in 3D by SELM we adjusted the confocal plane to correspond to different pit depth. SELM results for different imaging planes are shown in Figs. $2 d$ and e, respectively, clearly resolving stronger modes along the rims of the structures when the focal plane is positioned just above the surface plane. These angled trough edge modes also appear in simulations (Figs. $2 \mathrm{~h}$ and i). Strikingly, the simulations also predict a mode towards the bottom tip of the pit structure, corresponding to the strong mode seen experimentally in Fig. 2c. There has been a lively debate in the recent literature regarding the position of this mode, predicting it to occur either in the middle of the pit or towards the bottom apex of the pit. Clearly we observe the latter here, which is in accordance with our own simulations as shown in Figure $2 \mathrm{~h}^{19,29,30}$. The SELM technique proves directly the existence of a mode in close proximity to the bottom apex of the pyramidal pit, appearing as a highly confined region in Figures $2 \mathrm{c}$ and e. Less apparent in the simulations are the four angled edge modes that we observe experimentally along the trough diagonals. These modes are caused by imperfections in the pit structure that are likely to stem from the practical difficulty of depositing a metal coating uniformly across a sharp concave edge, resulting in momentum vector discontinuities for the generated plasmons, together with roughness. Both can give rise to a localized edge mode that is not captured by simulations. 
A further advantage of SELM is its wide-field imaging capability which allows the identification of interactions between different plasmonic structures. In the case of Klarite SELM confirms that there is no delocalization of plasmons between the neighboring pits.
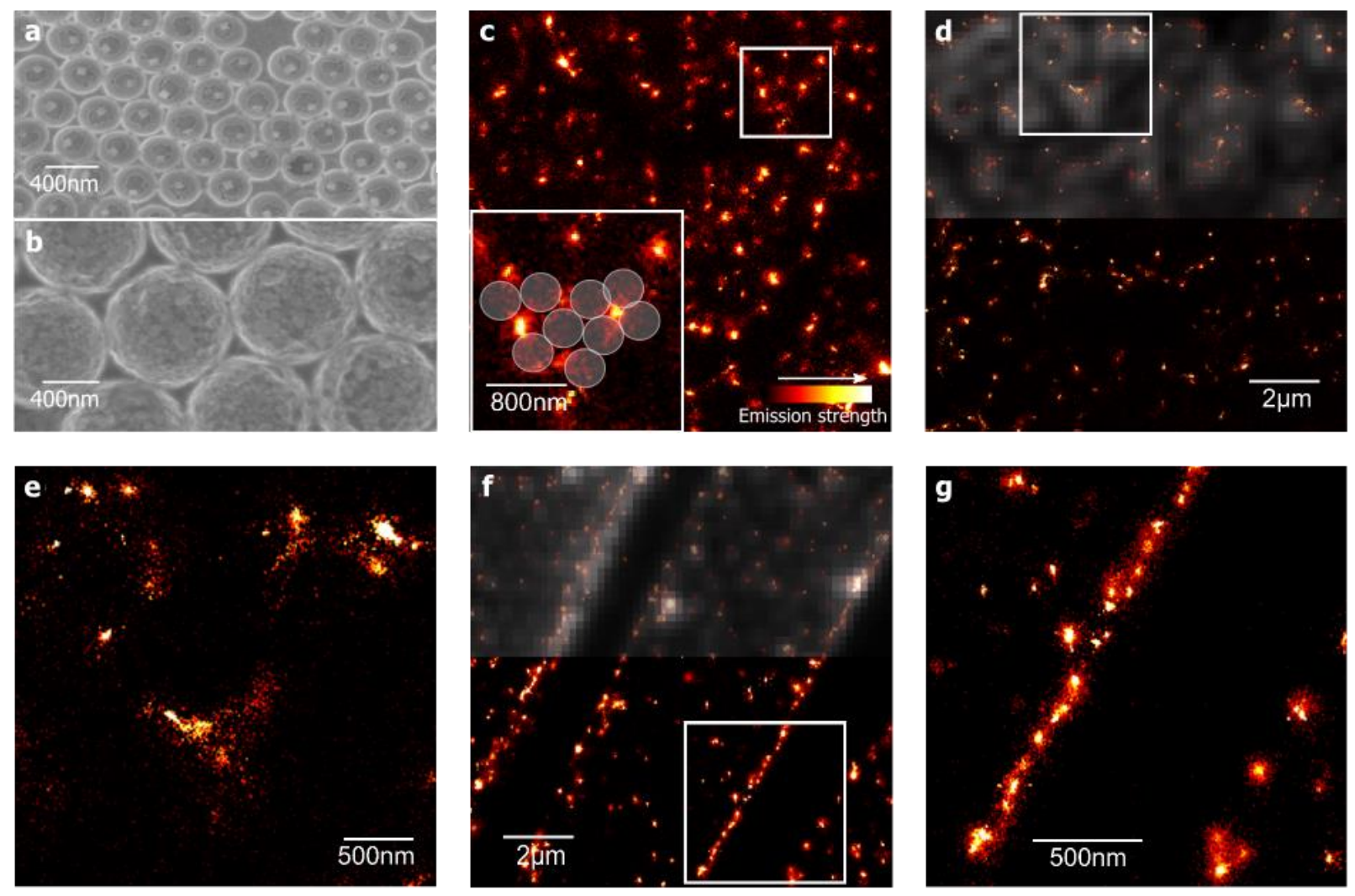

Figure 3: a, b) SEM images of $1 \mu \mathrm{m}$, and 400nm nanovoids voids respectively, the emission strength of individual localizations is color-coded. c) Highly resolved SELM image of $\mathrm{D}=400 \mathrm{~nm}$ nanovoids showing a granular distribution of individual highly active spots. Unlike Klarite, enhanced fields on this sample do not show a clear pattern. The inset shows an expanded view and the possible location of the voids such that the hotspots imaged by SELM are at their intersections. d) The SELM image of a labelled $D=1000 \mathrm{~nm}$ void sample reveals a less dense distribution of such hotspots. Often, two or more 'hotspots' are close together, as visible in the zoomed image in (e). f) Scratches on the surface (here $D=600 \mathrm{~nm}$ ) are clearly visible as dark, non-active areas. The edges of the scratch 
however show strong enhancements. (g) A zoomed in image of the scratch, inset in f, which reveals a high density of point-like spots along the edge in $\mathrm{f}$.

In contrast to Klarite, super resolved SELM images of nanovoid structures as shown in Figure 3a exhibit remarkable differences and only for very large diameters ( $D=1000 \mathrm{~nm}$, Figs. 3d and e) can the hotspots belonging to individual voids be distinguished. For $\mathrm{D}=1000 \mathrm{~nm}$ the interstitial ridges seem to support weak localized plasmons as apparent from the less intense and sparse SELM images. Individual hotspots are not well separated for void diameters of $D=400 \mathrm{~nm}$ and $\mathrm{D}=600 \mathrm{~nm}$ (Figs. $3 \mathrm{c}$ and $\mathrm{f}, \mathrm{g}$ ). Unlike Klarite, nanovoids are known to support delocalized plasmons propagating from one void to the next via the rim (edge) surface ${ }^{21}$. This 'rim' mode has been shown to be significantly involved in generating SERS ${ }^{21,31}$. Hence, it is highly likely that localization occurs at the intersecting rims of three neighboring nanovoids in the hexagonal lattice. The intense bright regions or 'hotspots' observed between neighboring voids pinpoint the high field strengths and highly-confined plasmons which give rise to strong SEF at the observed emission wavelength of the dyes. The plasmonic properties of nanovoids are easily tailored by varying the size of the sphere templates and their size ${ }^{32}$. The SEF enhancements observed are dependent on the plasmon resonances at both the excitation and emission wavelengths. For larger void diameters, the 'hotspots' appear less dense (Figs. $3 \mathrm{c}, \mathrm{f}, \mathrm{g}$ ) as the plasmon resonances redshift $^{21}$ and hence detune from the excitation and detection wavelengths used here. Interestingly, the scratch observed through the void surface shows a distinct topological boundary and generates highly localized spots of SEF, which are surprisingly strong and dense, but clearly resolved by SELM (Figs. 3f, g). Such images of patterned substrates such as Klarite and nanovoids as well as nanostructures such as line scratch edges demonstrate that SELM is a generic method suitable for imaging plasmon fields in nanoscopic detail without requirements 
for complex photoactivation methods or external switching agents. One could argue that the orientational variations of fluorophore dipoles might cause misinterpretation of the data in terms of plasmonic field distributions. However, the DNA constructs are minute compared to the dimensions of surface features, even those stemming from surface roughness. Thus molecules are unhindered in their ability to change orientation and thus we do not expect the dipole orientations to be a function of molecule location across the substrate. While the orientation movements may differ they will do so stochastically and should average out over the acquisition times (20 ms), orders of magnitude larger than reorientation timescales, and the many frames (up to 10,000$)$ acquired in this work. Thus the emission patterns given by SELM images represent averages over all permitted dipole orientations.

The simplicity of the SELM imaging method relies on the autonomous photon-blinking observed. Hence, this behavior was investigated further to establish whether quenching of the fluorophore (label) by the metal surface plays a role in the phenomenon. To test this, we varied the distance of the dye molecule, as shown schematically in figure 4 by using different complementary strands. Thus the labels were positioned at different heights above the surface by using the DNA as a scaffold (see also the supporting information). Image stacks of 4200 frames were processed for each type of fluorophore labeled strand and the number of localizations (blinking events) plotted versus linker length as well as the mean intensity of all individual localizations in a stack. The graph (Fig. 4b) demonstrates that it is the number of on-off blinking events which decreases with decreasing fluorophore-metal distance rather than the intensity of each fluorescence event. The slight decrease of the number of localizations for a dye-surface distance greater than $10 \mathrm{~nm}$ can be attributed to decreasing intensities of individual blinking events with distance from the surface. Hence, photon bursts from single molecules with the 
fluorophore located further away from the surface have a higher likelihood of being not recorded by the algorithm employed by the rapidSTORM software used to analyze the photon statistics. In other words, as soon as a molecule is in the on-state, it is fluorescent and the fluorescence is as strong as permitted by the local electric field. The closer the fluorescent tag approaches the surface however, the lower its probability to enter into an on-state. This leads us to suggest that the DNA strands (and/or the fluorescent labels attached to them) undergo orientational change (sway) on the surface due to Brownian motion, which has been observed previously ${ }^{33}, 34$. The molecules' tilt angles can change dynamically on the surface as in the elastic bending diffusion model developed previously ${ }^{35}$. Although we use low salt concentration (low ionic strength, high Debye length) and high $\mathrm{pH}(8.1)$ to increase the probability of upright orientations, such elastic bending could still pivot around the hexa-ethyleneglycol thiol linker used to anchor the dsDNA on the gold surface. The ultra-high sensitivity of SELM on plasmonic surfaces enables us to visualize such motions. This dynamic bending which moves the dye couples two phenomena: it changes the distance to the surface which varies 1) the strength of the optical field experienced, and 2) the propensity for quenching. The strength of the field decreases further away from the surface and quenching occurs only in close proximity to the surface as electrons can tunnel directly (non-radiative transfer) from the fluorophore to the metal. Thus for dyes attached closest to the surface, positional changes are the smallest with respect to the surface; therefore these will experience the greatest degree of quenching and thus have the lowest probability to be in a fluorescent on-state. Although quenching dominates the number of fluorescence events in this case, on occasions the fluorophore 'escapes the quenching regime', bursts of very high intensity are observed, because the plasmon field is strongest near the surface, leading to enhanced SEF. Moreover, due to the high field strengths associated with plasmons, dyes could be switched back 
into an on-state at any time without a chemical change of their environment. This would also explain the low rate of photo-bleaching observed (see Figure S7 supporting information). While the autonomous blinking behavior removes the complexity of using external agents or photoactivateable fluorophores, the distance dependence of the blinking frequency also opens up other possibilities. Thus SELM could be used in sensing and imaging of the 3D environment of molecules near metallic surfaces by coupling the distance-dependent frequency of blinking events to the hot-spot localization.
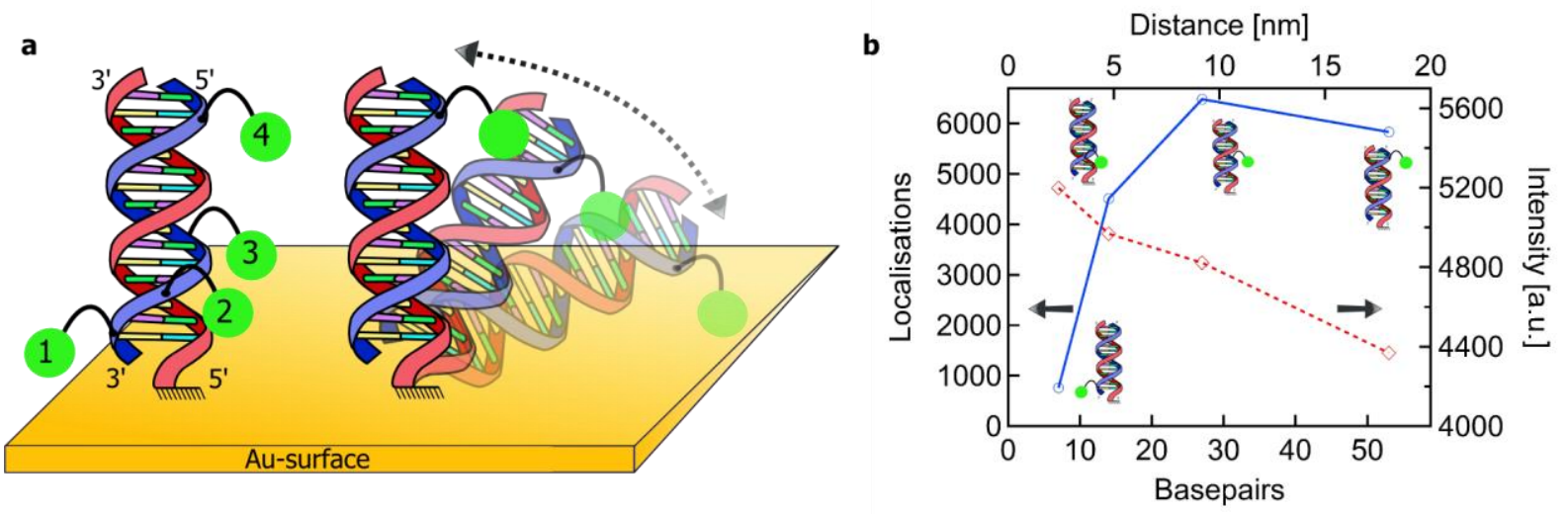

Figure 4: a) Schematic showing DNA used as a nanoscopic ruler to vary the distance of a dye above the surface. Elastic bending of DNA causes blinking. b) Localizations (number of blinking events) and their intensity, per 4200 frames vs. dye distance from the surface.

\section{Conclusion}

Surface enhanced localization microscopy (SELM) opens new possibilities to overcome conventional, diffraction-limited far-field scattering techniques to infer field distributions of nanoscopic plasmons. We have demonstrated the use of SELM for the direct visualization of plasmonic fields with nanometer resolution, which revealed plasmonic hotspots including those that arise from surface imperfections. SELM uses single molecule localization of surfaceenhanced fluorescence but without the use of photoactivation or exogenous switching methods. 
The autonomous switching of fluorescence in SELM was found to depend on the distance from the metallic surface, which can potentially be utilized for super-resolution microscopy in 3D. This work paves the way for uncomplicated super-resolution microscopy of metallic nanostructures, useful in a wide range of fields including plasmonics, surface-enhanced spectroscopies, electrochemistry and surface-science.

Acknowledgement:

The authors would like to thank Sebastian van de Linde and Eric Rees for their contributions to super-resolution imaging. We acknowledge financial support from EPSRC grant EP/G060649/1, EP/I012060/1, ERC grant LINASS 320503.

Author contributions:

CS performed all the experiments and analyzed all the results; ME implemented the setup and did all the simulations together with MC and GS; CFK and SM conceptualized and supervised the project; CFK, SM and JJB contributed to the materials, methods and techniques; CS, SM and CFK wrote the paper, all authors went to through the paper and contributed to the results and discussion.

Author Information:

Competing financial interests: The authors declare no competing financial interests. 
Correspondence and requests for materials should be addressed to (SM) s.mahajan@soton.ac.uk or (CFK) $\underline{\text { cfk23@cam.ac.uk }}$

\section{References}

1. $\quad$ Brongersma, M. L.; Shalaev, V. M. Science 2010, 328, 440-441.

2. Ozbay, E. Science 2006, 311, 189-193.

3. Homola, J.; Yee, S. S.; Gauglitz, G. Sens. Actuators, B 1999, 54, 3-15.

4. $\quad$ Barnes, W. L.; Dereux, A.; Ebbesen, T. W. Nature 2003, 424, 824-830.

5. $\quad$ Aroca, R. F. Phys. Chem. Chem. Phys. 2013, 15, 5355-5363.

6. $\quad$ Fleischmann, M.; Hendra, P.; McQuillan, A. Chem. Phys. Lett. 1974, 26, 163-166.

7. Zayats, A. V.; Smolyaninov, I. I.; Maradudin, A. A. Phys. Rep. 2005, 408, 131-314.

8. Nelayah, J.; Kociak, M.; Stephan, O.; Garcia de Abajo, F. J.; Tence, M.; Henrard, L.; Taverna, D.; Pastoriza-Santos, I.; Liz-Marzan, L. M.; Colliex, C. Nat. Phys. 2007, 3, 348-353.

9. $\quad$ Nicoletti, O.; de La Peña, F.; Leary, R. K.; Holland, D. J.; Ducati, C.; Midgley, P. A. Nature 2013, 502, 80-84.

10. $\quad$ Kneipp, K.; Wang, Y.; Kneipp, H.; Perelman, L. T.; Itzkan, I.; Dasari, R. R.; Feld, M. S. Phys. Rev. Lett. 1997, 78, 1667.

11. Brown, R. J.; Milton, M. J. J. Raman Spectrosc. 2008, 39, 1313-1326.

12. Goldstein, J.; Newbury, D. E.; Joy, D. C.; Lyman, C. E.; Echlin, P.; Lifshin, E.; Sawyer, L.; Michael, J. R., Scanning electron microscopy and X-ray microanalysis. Springer: 2003.

13. Hofmann, C. E.; Vesseur, E. J. R.; Sweatlock, L. A.; Lezec, H. J.; García de Abajo, F. J.; Polman, A.; Atwater, H. A. Nano Lett. 2007, 7, 3612-3617.

14. Barnes, W. J. Opt. A: Pure Appl. Opt. 2009, 11, 114002.

15. Rees, E. J.; Erdelyi, M.; Pinotsi, D.; Knight, A.; Metcalf, D.; Kaminski, C. F. Optical Nanoscopy 2012, 1, 1-10.

16. Rust, M. J.; Bates, M.; Zhuang, X. Nat. Methods 2006, 3, 793-796.

17. Heilemann, M.; van de Linde, S.; Schüttpelz, M.; Kasper, R.; Seefeldt, B.; Mukherjee, A.; Tinnefeld, P.; Sauer, M. Angew. Chem., Int. Ed. 2008, 47, 6172-6176.

18. Lin, H.; Centeno, S. P.; Su, L.; Kenens, B.; Rocha, S.; Sliwa, M.; Hofkens, J.; Uji-i, H. ChemPhysChem 2012, 13, 973-981.

19. Perney, N.; de Abajo, F. G.; Baumberg, J.; Tang, A.; Netti, M.; Charlton, M.; Zoorob, M. Phys. Rev. B 2007, 76, 035426.

20. Baumberg, J. J.; Kelf, T. A.; Sugawara, Y.; Cintra, S.; Abdelsalam, M. E.; Bartlett, P. N.; Russell, A. E. Nano Lett. 2005, 5, 2262-2267.

21. Cole, R. M.; Baumberg, J. J.; García de Abajo, F. J.; Mahajan, S.; Abdelsalam, M.; Bartlett, P. N. Nano Lett. 2007, 7, 2094-2100.

22. Mahajan, S.; Cole, R. M.; Soares, B. F.; Pelfrey, S. H.; Russell, A. E.; Baumberg, J. J.; Bartlett, P. N. J. Phys. Chem. C 2009, 113, 9284-9289.

23. Steuwe, C.; Kaminski, C. F.; Baumberg, J. J.; Mahajan, S. Nano Lett. 2011, 11, 53395343.

24. Lakowicz, J. R. Anal. Biochem. 2001, 298, 1-24.

25. Anger, P.; Bharadwaj, P.; Novotny, L. Phys. Rev. Lett. 2006, 96, 113002. 
26. Rees, E. J.; Erdelyi, M.; Schierle, G. S. K.; Knight, A.; Kaminski, C. F. J. Opt. (Bristol, U. K.) 2013, 15, 094012.

27. van de Linde, S.; Kasper, R.; Heilemann, M.; Sauer, M. Appl. Phys. B: Lasers Opt. 2008, 93, 725-731.

28. Gersten, J.; Nitzan, A. J. Chem. Phys. 2008, 73, 3023-3037.

29. Vernon, K.; Davis, T.; Scholes, F.; Gomez, D.; Lau, D. J. Raman Spectrosc. 2010, 41, 1106-1111.

30. Mechler, M.; Kukhlevsky, S. V.; Mechler, A.; McNaughton, D. Phys. Chem. Chem. Phys. 2011, 13, 20772-20778.

31. Mahajan, S.; Abdelsalam, M.; Suguwara, Y.; Cintra, S.; Russell, A.; Baumberg, J.; Bartlett, P. Phys. Chem. Chem. Phys. 2007, 9, 104-109.

32. Cintra, S.; Abdelsalam, M. E.; Bartlett, P. N.; Baumberg, J. J.; Kelf, T. A.; Sugawara, Y.; Russell, A. E. Faraday Discuss. 2006, 132, 191-199.

33. Chan, V.; Graves, D. J.; Fortina, P.; McKenzie, S. E. Langmuir 1997, 13, 320-329.

34. Rant, U.; Arinaga, K.; Fujita, S.; Yokoyama, N.; Abstreiter, G.; Tornow, M. Nano Lett. 2004, 4, 2441-2445.

35. Anne, A.; Demaille, C. J. Am. Chem. Soc. 2006, 128, 542-557. 